\title{
Propuesta de un sistema automatizado para la obtención de sal en Azuero, República de Panamá
}

\section{Proposal for an automated system for obtaining salt in Azuero, Republic of Panama}

\author{
Paola Fuentes ${ }^{l}$, Lissette Peña ${ }^{1}$, Omar Sánchez ${ }^{2}$, Yessica Sáez ${ }^{3 *}$ \\ ${ }^{1}$ Licenciatura en Ingeniería Electrónica y Telecomunicaciones-Universidad Tecnológica de Panamá (Sede Azuero) \\ ${ }^{2}$ Licenciatura en Ingeniería Eléctrica y Electrónica- Universidad Tecnológica de Panamá (Sede Azuero) \\ ${ }^{3}$ Facultad de Ingeniería Eléctrica-Universidad Tecnológica de Panamá (Sede Azuero)
}

\begin{abstract}
Resumen En la República de Panamá, el proceso de obtención de sal emplea la mano de obra humana, careciendo de muchos avances tecnológicos, lo que influye de manera directa y sumada a otras variables, la cantidad y calidad del mineral que se llegue a producir. El objetivo principal de esta investigación es proponer un sistema remoto que le permita a los diferentes dueños y trabajadores de salinas, monitorear y controlar diferentes procesos necesarios para la producción de sal. Para la realización de esta investigación, se procedió a recolectar información sobre el proceso de producción de sal, mediante la aplicación de cuestionarios y entrevistas realizadas a las distintas personas que laboran en la Federación Nacional de Cooperativas Salineras de Panamá, R.L., así como datos y documentos facilitados que permitieron obtener una visión amplia del proceso realizado para la obtención de sal. Los resultados de estos cuestionarios muestran que Panamá produce su propia sal para el consumo nacional, siendo Azuero la región donde la producción de sal se da con mayor auge y es por ello que basamos nuestra investigación en esta zona específica. Gracias a las respuestas obtenidas de los cuestionarios aplicados a los salineros, de nuestra región de estudio, se propone un sistema automatizado utilizando un controlador lógico programable (PLC) que permitirá la medición del nivel del mar en puntos específicos con la ayuda de sensores especializados. Además de lo anterior, el sistema propuesto implementará señales GSM con el fin de informar al personal encargado de la salina sobre el estado en que se encuentra el sistema.
\end{abstract}

Palabras clave Automatización, producción de sal, salinas, tecnología.

\begin{abstract}
In the Republic of Panama, the process of obtaining salt uses human manpower, lacking many technological advances, which directly influences and in addition to other variables, the quantity and quality of the mineral that is produced. The main objective of this research is to propose a remote system that allows the different owners and workers of salinas to monitor and control different processes, necessary for the production of salt. To carry out this research, we proceeded to collect information on the salt production process, through the application of questionnaires and interviews with the different people who work in the National Federation of Salinas Cooperatives of Panama R.L., as well as data and documents provided that allowed us a broad view of the process carried out to obtain salt. The results of these questionnaires show that Panama produces its own salt for national consumption, being Azuero the region where salt it is produce the most and that is why we based our research on this specific area. Thanks to the responses obtained from the questionnaires applied to the salineros in our study region, an automated system is proposed using a programmable logic controller (PLC) that will allow the measurement of sea level at specific points with the help of specialized sensors. in addition to the above, the proposed system will implement GSM signals in order to inform the personnel in charge of the saline about the state of the system.
\end{abstract}

Keywords Automation, salt production, salt works, technology.

* Corresponding author: yessica.saez@utp.ac.pa

\section{Introducción}

Las salinas panameñas, típicamente pequeñas empresas familiares con recursos económicos limitados, han sido muy conservadoras en cuanto a la implementación de tecnología. El proceso tradicional de producción de sal empleado actualmente en nuestro país se basa en la recolección de agua de mar y en un entorno adecuado para la evaporación solar natural. Esta técnica depende en gran medida de la temperatura diaria y los 
vientos predominantes para promover la evaporación, por lo que es común y comercialmente viable en las zonas costeras. Dicho sistema pasivo, simple, con poco avance tecnológico, donde la intervención humana juega un papel crucial, se ha utilizado por siglos [1]. Como resultado, el período de producción de sal está limitado por las condiciones meteorológicas que promueven las condiciones ideales como: altas temperaturas diarias, humedad, vientos entre otros, algo que no ocurre exactamente todos los días.

La producción de sal a nivel mundial se ha visto afectada en los últimos años debido al cambio climático, mientras que la demanda de este mineral sigue en aumento, producto del incremento de la población, pues el mismo no solo juega un papel importante en varios campos e industrias como procesamiento de metales, cerámica, petroquímica, industria textil, etc., sino que además el cuerpo humano / animal requiere una ingesta adecuada de sal para asegurar su crecimiento, sistemas inmunes y reproductivos fuertes [1]. La falta de sodio en las personas puede traer grandes consecuencias, provocando una deficiencia en la génesis de los impulsos eléctricos en las neuronas, lo que deriva problemas como letargo, desorientación, debilidad muscular, convulsiones, coma y lo que es peor, la muerte [2].

Entre los años 2006 y 2014 se experimentó un incremento en la demanda de sal del $45 \%$, atribuido principalmente al

crecimiento demográfico [3]. Hasta el año 2015 los mayores productores de sal eran Estados Unidos, China e India. En la figura 1 se muestra el ranking de los países con mayor producción de sal medidos en miles de tonelada [4].

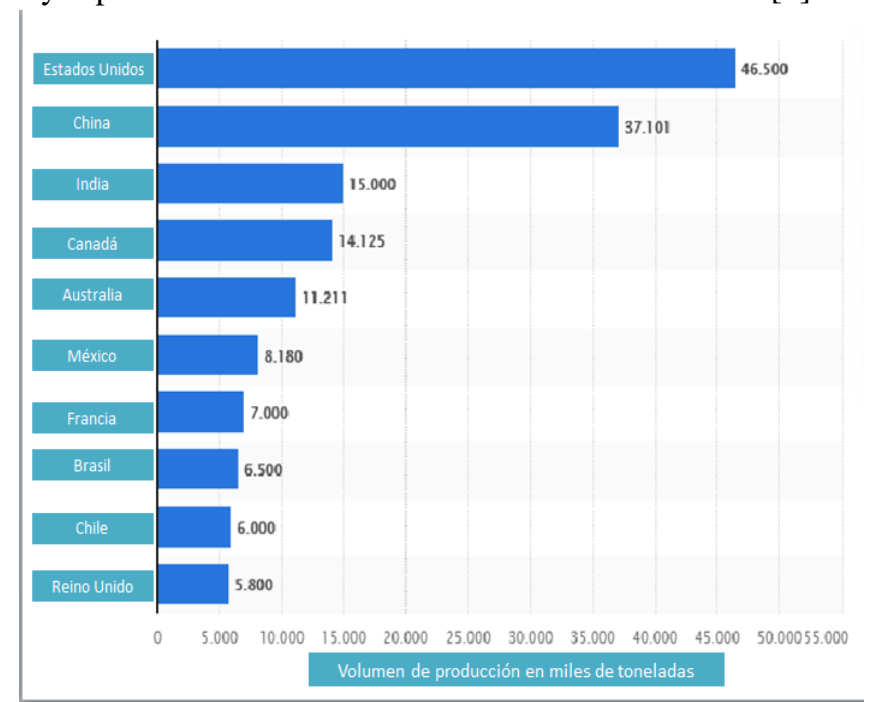

Figura 1. Ranking de los países con mayor producción de sal en el 2015. Fuente: Statista [4]
Según la información de la figura 1, se aprecia que México es el país con mayor producción de sal en Latinoamérica y en el cual se encuentra la exportadora de sal más grande del mundo [5].

En la literatura se han propuesto diversas soluciones para mejorar el proceso de obtención de sal y como consecuencia, aumentar la producción de la misma. Un ejemplo de lo anterior fue un estudio realizado en España, el cual buscó implementar colectores cilíndricos parabólicos e intercambiadores de calor a las salinas tradicionales, lo que permitiría aumentar las tasas de producción [1]. Los autores se basaron en el hecho de que la producción de este mineral es mayor durante los meses de verano. En la actualidad existen empresas como Grupo Industrial Roche, ubicada en Yucatán México, quienes decidieron implementar tecnología en su planta, lo que les ha permitido aumentar su producción de 5 mil toneladas anuales, las cuales eran obtenidas de manera tradicional a $750 \mathrm{mil}$ toneladas anuales mediante el uso de diversos mecanismos tecnológicos [6].

La tecnología avanzada también está disponible para la producción de sal, pero la instalación de la planta y el costo del equipo se estima que es muy alto. Por ejemplo, países con alta humedad han dedicado sus esfuerzos al uso de avances tecnológicos para producir sal a una humedad elevada, ya que a medida que esta aumenta, el proceso de producción se dificulta. Según la entrevista realizada a la Federación Nacional de Cooperativas Salineras de Panamá, R.L. (FENCOSPA, R.L), hace un par de años atrás, un holandés realizó estudios demostrando que se podría producir sal con una humedad de $2.34 \%$. En este estudio se llegó a la conclusión de que para llevar a cabo dicho proceso se debe invertir una mayor cantidad de dinero y realizar mejoras a las diferentes salinas. Esto es muy interesante para nuestro país, ya que debemos considerar que una de las principales problemáticas en nuestra producción de sal es el alto grado de humedad que tenemos, provocando que la sal se obtenga con una humedad de alrededor del $8 \%$, en comparación con otros países que la producen a un promedio de $1.5 \%$.

La producción de sal en la Región azuerence de la República de Panamá se ha visto mermada no solo por el cambio climático, sino también por el uso de un método tradicional de evaporación solar en el que la mayoría de las operaciones son manejadas por los trabajadores de sal manualmente y que ha causado la deserción de muchos salineros. Esto se debe a que la fabricación de sal se realiza de manera tradicional, todas las operaciones como el llenado de agua en el estanque, el mantenimiento del nivel de agua, el nivel de salinidad del agua, la inyección de agua dulce, etc. son manejadas por los trabajadores, la figura 2 muestra la cantidad de sal obtenida anualmente desde el año 2000 hasta el 2017. 


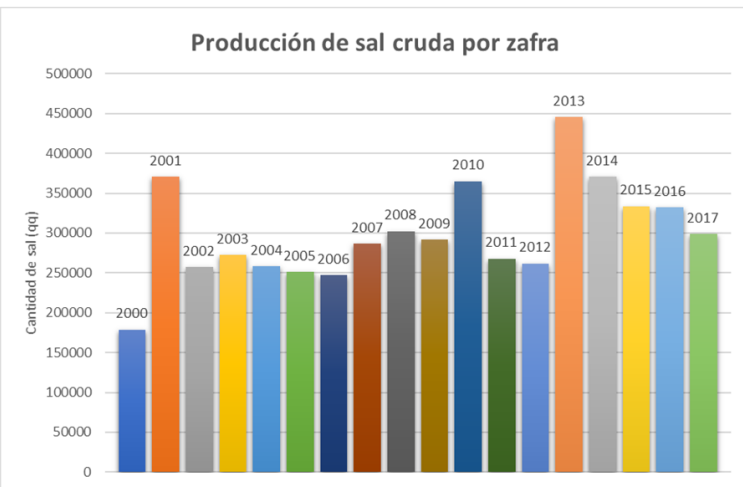

Figura 2. Producción de sal en Panamá desde el año 2000 hasta el 2017. Fuente: FENCOSPA, R.L. [7].

En la figura 2 se puede notar la baja que ha tenido la producción de este mineral desde el año 2014 hasta el año 2017. Es por ello que el objetivo principal de esta investigación es proponer un sistema remoto que le permita a los diferentes dueños y trabajadores de salinas, monitorear y controlar mediante la utilización de un control lógico programable (PLC), operaciones como la medición del nivel del mar en los puntos especificados con la ayuda de sensores especializados, los cuales se encontrarán especificados en la sección de materiales y métodos. Para la transmisión de las señales monitoreadas, el PLC a través de una señal GSM, le hará saber a quién esté a cargo de la salina, cualquier estado en que se encuentre el sistema, además del control de la misma si es necesario.

Este artículo se encuentra organizado de la siguiente manera: la sección 2 muestra los antecedentes. La sección 3 describe los diferentes materiales y métodos utilizados. En la sección 4 se encontrarán los resultados de las encuestas aplicadas a los diferentes salineros. La sección 5 explica el diseño conceptual propuesto y en la sección 6 se encontrarán las discusiones.

\section{Antecedentes}

En Panamá la producción de sal se realiza aprovechando los aguajes (mareas más altas que el nivel normal). Desde la llegada de los españoles se construyeron canales para captar el agua de esas mareas, dándole un manejo para producir sal. A este antiguo sistema se le conoce como "destajos de tierra" (fosas en las que se vierte el agua salada) [5]. En tiempos de aguaje, el agua entra por los esteros (terrenos pantanosos) y se desplaza por las albinas (tierra con cierto contenido de sal), las cuales quedan inundadas por dichas aguas, quedando estancadas en pequeñas cantidades en donde, por efecto de los rayos solares, parte de esta se evapora formando cristales que se precipitan en el fondo de fosas o destajos.

En Panamá las regiones que se dedican a esta actividad son la región azuerence y la provincia de Coclé. En la región de Azuero se encuentra la Cooperativa Reyes Espino, R.L. ubicada en Guararé, la Cooperativa Salineros Santeños, R.L. ubicada en La Villa de Los Santos, la Cooperativa Sal Sarigua,
R.L. ubicada en Chitré y en la provincia de Coclé se ubica Cooperativa Marín Campos, R.L. específicamente en Aguadulce [8].

Para esta investigación estaremos trabajando con la Cooperativa Salineros Santeños, R.L. La figura 3 muestra el mapa de ubicación exacta de las salinas a trabajar.

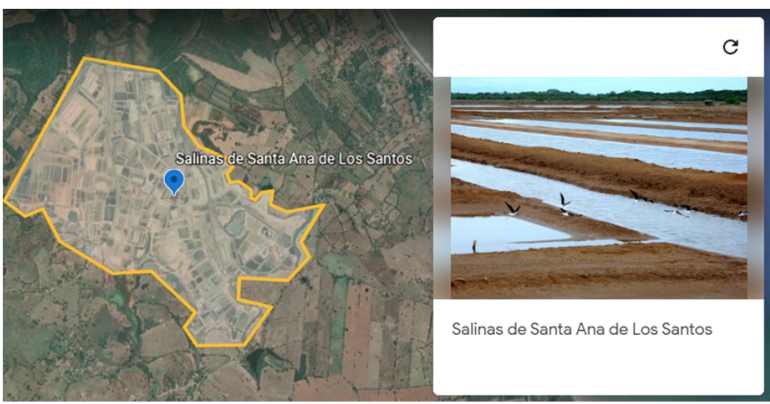

Figura 3 Salinas de Santa Ana de Los Santos.

Fuente: Google Earth. [9].

Por motivos de calidad y de cantidad, la actividad salinera ha evolucionado y se ha transformado en un sistema con técnicas nuevas y un tanto modernas, aunque la implementación de tecnología más avanzada pudiera permitir obtener una mayor producción de sal. En la actualidad los salineros siguen utilizando las mismas instalaciones tradicionales, pero han logrado instalar mejoras como: compuertas, construcción de lagos y la más significativa; el reemplazo de destajos de tierra a carpetas o cristalizadores de plástico, lo que han permitido obtener una sal más limpia, aumentar el almacenamiento de agua y la producción del mineral. La figura 4 ilustra el proceso actual utilizado en las salinas.

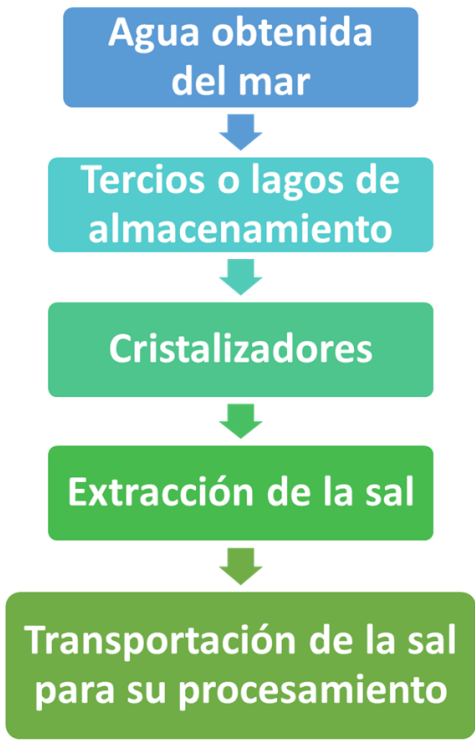

Figura 4. Proceso actualmente utilizado para la obtención de sal. Fuente: Entrevista a salineros. 
El proceso actual de producción de sal inicia con la captación del agua aprovechando las altas mareas, momento en el cual el agua contiene de 1 a 1.5 grados de salinidad. Esta agua va pasando a los lagos de reserva o "tercios" a través de compuertas pequeñas, permitiendo que el sol y el viento aceleren la evaporación, dando como resultado el aumento de la concentración de la sal. Esta agua permanece retenida en los tercios por un periodo aproximado de 15 días, hasta obtener un nivel de pre-cristalización de 18 grados o más, según la escala Baumé (escala utilizada en la medida de las concentraciones de ciertas soluciones, originalmente se basa en la salinidad del agua y salmuera) 6 . Una vez se cumple este proceso se procede a bombear hacia los distintos cristalizadores, destajos o tinas plásticas, donde iniciará la precipitación de los cristales de sal hasta lograr alcanzar los 24-26 grados. Este proceso puede durar de 4 a 6 días dependiendo de la intensidad del sol y la cantidad de agua depositada en las tinas. Es importante mencionar que las tinas deben llegar a tener un espesor de 3 a 4 pulgadas. Una vez cristalizada la sal, se comienza la extracción o cosecha, la cual se suele realizar con instrumentos de madera o plástico como cepillos, escobillones u otra herramienta similar. Se amontona la sal en la tina, se deja escurrir, se extrae y se le da un reposo de 48 horas para ser depositada en los diferentes sacos y luego se traslada y procesada para su posterior comercialización [10].

Es importante mencionar que todo el proceso, desde la captación del agua hasta que la sal se traslada para ser procesada es totalmente manual, lo que de cierta manera impide el incremento de la producción. Una de las principales desventajas para los salineros o personas encargadas, es el momento de la captación del agua, ya que como se ha mencionado anteriormente estas depende de las altas mareas, por ende se debe estar en constante vigilancia y una vez suba la marea, sin importar la hora en que se de este fenómeno natural, las personas deben trasladarse a la salina para abrir las compuertas y dejar pasar el agua a los tercios hasta que los tercios alcancen el nivel requerido, es importante mencionar que el nivel de los tercios puede variar, dependiendo de la salina. Es por ello que la implementación de un sistema más moderno podría ayudar a los diferentes salineros, evitando trasladarse a las salinas durante horas de la madrugada y así los mismos puedan tener un mayor desempeño de sus funciones durante las labores diurnas en la salina.

\section{Materiales y métodos}

\subsection{Materiales}

Teniendo en cuenta el arduo trabajo de los salineros en sus labores, tanto al recolectar el agua de mar y el antiguo sistema que se utiliza para la producción de sal, se busca proponer la aplicación de tecnología que faciliten su labor, utilizando los siguientes equipos:

- Bomba centrífuga

- Disyuntor termomagnético
- Boyas

- Contactor

- Pulsador normalmente abierto

- Conmutador biestable

- Control lógico programable, EXM-8AC-R.

\subsection{Metodología}

Antes de realizar una propuesta de este tipo, se procedió a entrevistar al gerente de FENCOSPA, R.L., la participación de esta empresa se limita a las cooperativas asociadas que realizan la actividad de producción y mercadeo de sal, donde dos de ellas son productoras. Se han escogido las mismas ya que actualmente Azuero es la región del país que más sal produce, razón por la cual este estudio se realiza específicamente en el corregimiento de Santa Ana, provincia de Los Santos [11]. Obtuvimos información estadística interesante relacionada con las diferentes cooperativas que están afiliadas a la federación. Por ejemplo, dentro de la federación existen un total de 173 productores de sal. Estos datos sentaron las bases para el diseño de una encuesta que básicamente buscaba medir el grado de aceptación de los salineros con respecto al uso de tecnología en el proceso de producción de sal.

Se tomó en cuenta la cantidad de productores pertenecientes a la Cooperativa Salineros Santeños, que cuenta con 93 productores [8], de ahí se extrajo una muestra de 75 utilizando la formula (1) obtenida de [12]:

$$
n=\frac{Z^{2} P Q N}{E^{2}(N-1)+P Q Z^{2}}
$$

donde $\mathrm{Z}$ es el valor obtenido mediante niveles de confianza (en este caso se utilizó un grado de confiabilidad de $95 \%$ para el cual $\mathrm{Z}=1.96$ ). El producto $\mathrm{PQ}$ representa la varianza de la población, el cual, al ser desconocido se utilizó $\mathrm{PQ}=0.25$, se estimó un grado de error del $5 \%$ y $\mathrm{N}$ es el tamaño de la población en estudio.

Luego se procedió a visitar las diferentes salinas ubicadas en el corregimiento de Santa Ana, provincia de Los Santos, con el objetivo de conocer más sobre el proceso de la obtención de la materia prima y así indagar sobre su opinión acerca del uso de tecnología en el proceso de producción de sal. Se aplicó la encuesta a la muestra determinada con el propósito de conocer la opinión de los trabajadores acerca del sistema antiguo y sus sugerencias en caso de querer modernizar el mecanismo utilizado. Además, se incluyó en dicha encuesta, una descripción de un sistema propuesto por nosotros para así conocer el grado de aceptación de los trabajadores hacia un sistema con mayor tecnología. Las preguntas de la encuesta fueron las siguientes:

- ¿Cómo se siente con el sistema utilizado actualmente?

- ¿Piensa que se debe cambiar el sistema?

- ¿Cómo considera el sistema propuesto? 


\section{Resultados}

A continuación, se muestran los resultados de la encuesta aplicada a los salineros, así como el diseño conceptual del sistema propuesto.

\subsection{Resultados de las encuestas}

Mediante la encuesta aplicada a los distintos productores de sal pudimos obtener un referente a su grado de aceptación con respecto al uso de la tecnología en el control y automatización del sistema utilizado actualmente en las salinas. A continuación, se muestran algunas de las preguntas realizadas con sus diferentes opciones.

Un $75 \%$ de las personas encuestadas dijo sentirse bien con el sistema utilizado actualmente mientras que un $25 \%$ no se siente bien, debido a que es un trabajo muy pesado $\mathrm{y}$ rudimentario (ver gráfica 1 ).

¿CÓMO SE SIENTE CON EL SISTEMA UTILIZADO ACTUALMENTE?

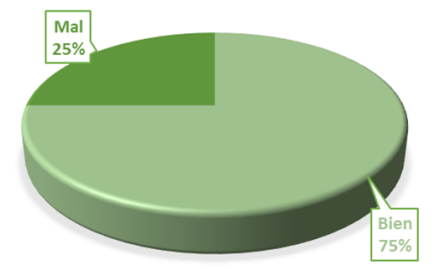

Gráfica 1. ¿Cómo se siente con el sistema utilizado actualmente?

Con respecto a la posibilidad de añadir cambios al sistema actual utilizado, un $60 \%$ está de acuerdo, algunos sugieren que se apliquen nuevas tecnologías que no sean tan costosas y que mejoren la calidad y cantidad de la producción, sin embargo, un $40 \%$ dice que se debe dejar el sistema actual tal como está, ya que es una tradición de muchos años, y prefieren "algo bueno conocido que malo por conocer" (ver grafica 2 ).

¿PIENSA QUE SE DEBE CAMBIAR EL SISTEMA?

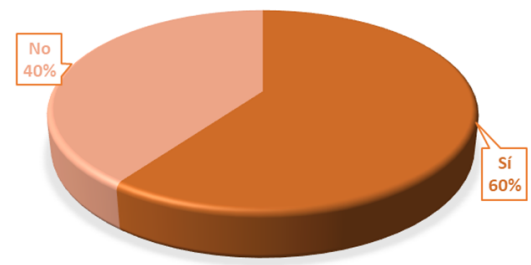

Gráfica 2. ¿Piensa que se debe cambiar el sistema?

Basados en los resultados de las encuestas y las sugerencias de los trabajadores de las salinas, se procedió a realizar un diseño conceptual de un sistema cuyo objetivo principal es reducir las horas de trabajo constantes y mejorar la producción de sal. Se les mostró este diseño a los encuestados, en donde un $52.5 \%$ dijo que el sistema propuesto parece ser bueno, un $35 \%$ expresó que es regular y un $12.5 \%$ manifestó que es una excelente alternativa para mejorar el sistema (ver grafica 3 ).

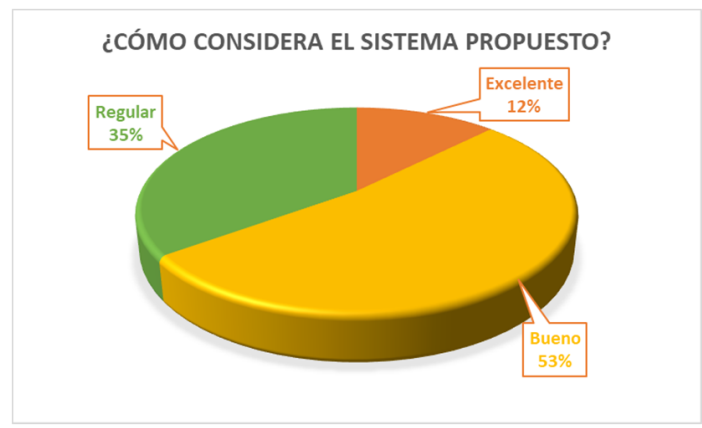

Gráfica 3. ¿Cómo considera el sistema propuesto?

\subsection{Diseño conceptual}

El propósito principal del sistema propuesto es brindar la facilidad de control y seguimiento, de manera remota y local, de las salinas ubicadas en nuestro país. Actualmente el procedimiento se hace a través de bombas comerciales de combustible, por lo que el control de las mismas es complicado, ya que los inyectores electrónicos que las mismas traen tienen su diseño de control interno. Por esta razón, se ha pensado en desarrollar el prototipo en función de bombas que trabajen con motores eléctricos.

\subsubsection{Diseño del prototipo}

Para el diseño del prototipo se utilizaron los materiales mencionados en la sección 3. En el punto más cercano al sistema de red eléctrica, se armará un panel de control donde, como primer componente, se colocará un disyuntor termomagnético para proteger el PLC, bombas y sensores, ante cualquier tipo de falla proveniente de la red (sobrecargas, fallas en las líneas, cortocircuito, calentamientos excesivos, entre otros). Bajo este dispositivo de protección se conectará un pulsador normalmente abierto. La cantidad dependerá del número de bombas ubicadas en la salina. Al utilizar estos pulsadores estamos dándole la oportunidad a los propietarios de las salinas de manejar el sistema manualmente, al conectar la salida directamente a los contactores. Para el modo automático, se colocará entre la boya y la entrada del PLC, un conmutador biestable. De manera que se pueda controlar tanto la comunicación entre el sensor y el PLC como el apagado de las bombas. Este último proceso se configurará dentro de la lógica. En las salidas del PLC se conectarán los contactores; al igual que con los conmutadores, su número dependerá de la cantidad de bombas.

Para el control del sistema automatizado, se colocarán boyas que funcionarán como medidores del nivel del agua. Las boyas se conectarán a contactos N.A, y estos a las entradas del PLC. Al subir la marea y llenarse el canal principal, el PLC 
recibirá este dato y enviará el mandato a la(s) bomba(s) ubicada(s) en el o los, lagos de reserva para que inicien a llenar el espacio físico. Las bombas deben ir conectadas al contactor. Se llenarán con agua hasta que las boyas, ubicadas ahí, lleguen hasta el máximo establecido. En este momento el PLC recibirá la señal de la boya y actuará sobre la bomba apagándola. Este proceso también se llevará a cabo en los "tercios" (lagos de reserva que están más cerca de los destajos de plástico, donde el agua alcanza el grado de salinidad adecuado más rápido) (ver figura 5)

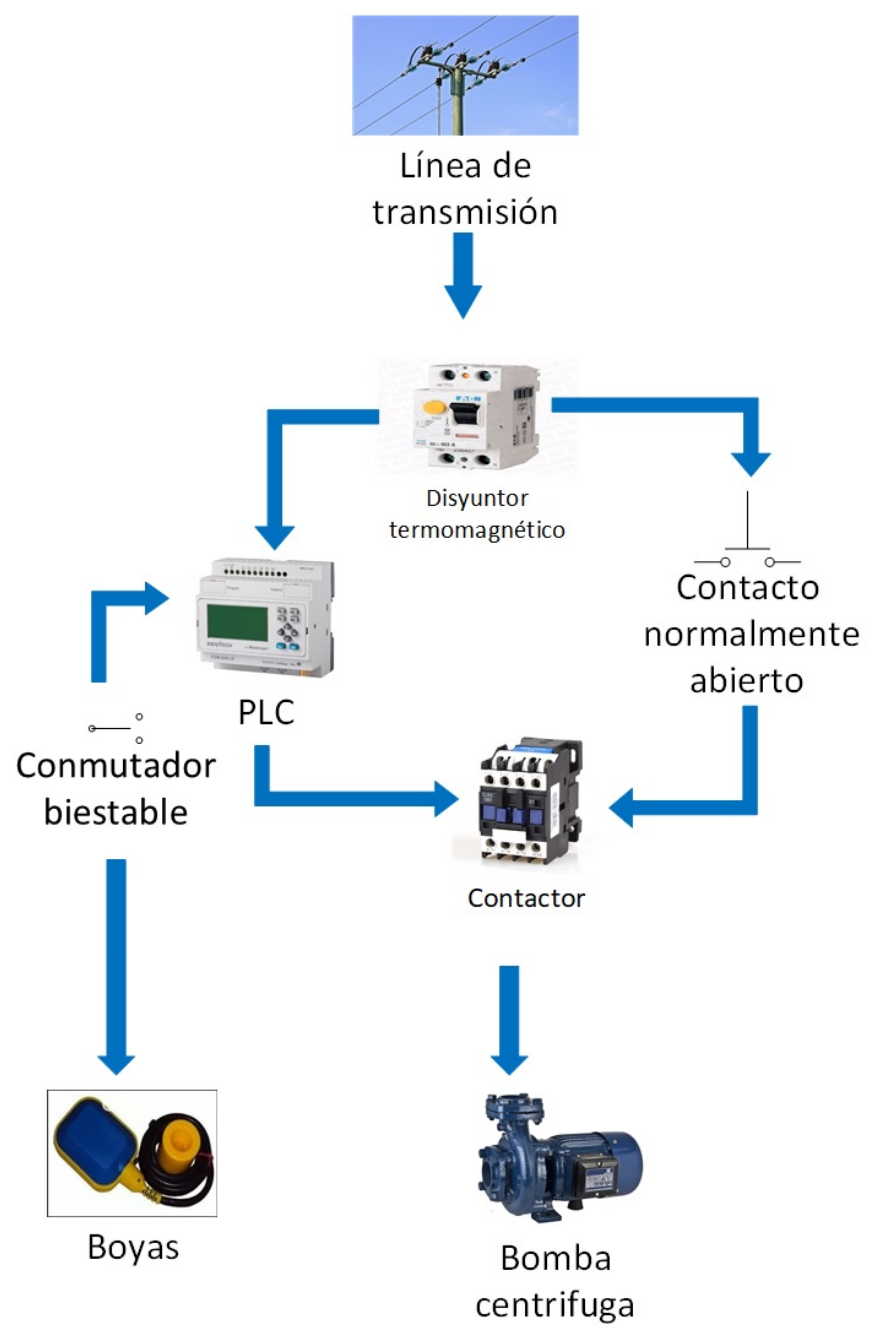

Figura 5. Esquemático del sistema propuesto.

Todo el proceso antes mencionado se logrará visualizar de forma remota, ya que el PLC cuenta con un módulo GSM incluido, por lo tanto, el prototipo contará con la disponibilidad de no solo monitorear sino de controlar desde cualquier lugar que tenga cobertura de una empresa telefónica.

No es necesario automatizar el control de calidad de la sal en términos de medir la salinidad con sensores. Este trabajo se le puede dejar a los encargados de las salinas. Una vez determinado el grado de salinidad necesario, a través del modo manual se pasará a llenar los destajos.

\subsubsection{Diseño del sistema de comunicación}

Cada uno de los sensores (boyas) se encargan de recoger las diferentes señales sobre el nivel del agua, las cuales una vez obtenidas, serán enviados al PLC y este por medio de una señal GSM las enviará a una plataforma, donde solamente el dueño o el personal encargado podrá tener acceso.

La plataforma recibirá los datos leídos por las boyas, dando parte a quien controla la salina de que a través de SMS o la misma aplicación, manipular en tiempo real funciones como el encendido o apagado de las bombas. Otra información que le será dispensada al usuario será en caso de algún error, ya sea problemas de algún sensor o bomba.

Esta aplicación es proporcionada por el mismo PLC por lo que no trae gran dificultad instalarlo, y por último los usuarios no deberán tener destreza de programación para parametrizar cualquier aspecto (ver figura 6).

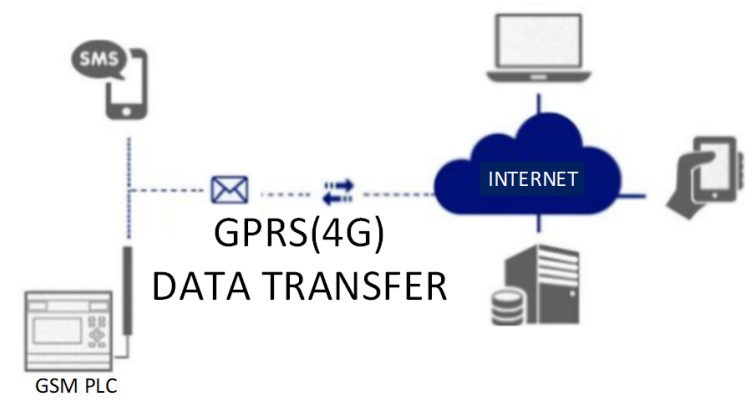

Figura 6. Esquemático del sistema de comunicación.

\section{Discusión}

Es importante destacar que, hasta la fecha, Panamá es un país que produce su propia sal para el consumo nacional y que Azuero es la región donde la producción de sal se da con mayor auge, sin embargo, el sistema utilizado actualmente presenta fallas debido a que se realiza de una manera manual, que a futuro junto con el cambio climático podría provocar escases del mineral y que el país tenga que exportarlo.

En nuestra visita a FENCOSPA, R.L, se conversó sobre dos proyectos propuestos para dar solución a problemáticas presentadas. El primero pretendía solucionar lo relacionado al tiempo que le toma a la sal cristalizarse. Este proyecto solo llegó a una fase inicial, ya que continuar con el nuevo sistema propuesto resultó ser de un alto costo y la federación no contaba con el presupuesto para continuarlo. El segundo es un proyecto que realizó un propietario de una salina. Este consiste en paneles solares que les proporciona energía a los motores para dejar de utilizar combustible. Estas dos propuestas demuestran que se han estado buscando alternativas para mejorar el sistema de producción de sal, pero que todavía hay mucho trabajo por realizar. 
Los resultados de las encuestas muestran que más de la mitad de los salineros están de acuerdo con que se deben aplicar cambios al sistema actual. Esto debido a que las nuevas generaciones no desean participar o seguir la tradición de cómo se produce la sal actualmente por ser un trabajo muy pesado. El sistema propuesto tuvo gran aceptación por los salineros y nos hizo referencia que el sistema suena bien, ya que solo se está automatizando parte del trabajo. Cabe mencionar, que el trabajo realizado en salinas es un trabajo hecho en conjunto, en otras palabras, un grupo de trabajadores contratados por el propietario de la salina. Por lo tanto, el hecho de automatizar todo, en general, traería como consecuencia una reducción de personal, por lo que esto pudo haber influido en su respuesta.

Finalmente, el sistema presentado en este trabajo es solo una propuesta conceptual. Como trabajo futuro, se espera llevarlo a prueba para comprobar su funcionamiento, de manera que el sistema sea óptimo. Igualmente se espera realizar más investigaciones en el área, con el fin de encontrar respuestas que sean tal vez menos costosas o más sencillas para el beneficio de los salineros.

\section{AGRADECIMIENTOS}

Se le agradece al gerente de FENCOSPA, R.L., Mgtr. Modesto Delgado y a sus colaboradores, a los salineros del corregimiento de Santa Ana de la provincia de Los Santos, Panamá, por el apoyo, información y la ayuda brindada.

\section{REFERENCIAS}

[1] Flagg Díaz, Robert \& Stewart y Susan \& Brownson, Je, «Use of Concentrated Solar Thermal Energy System to Enhance Sea Salt Production in Southern Spain,» 2012.

[2] F. M. B., «La sal es indispensable para la vida, pero ¿cuánta?,» Revista Chilena de nutrición, vol. 39, nº 4, pp. 192-195, 2012.

[3] Statista Research Department, «Statista,» 6 Agosto 2015. [En línea].

Available: https://es.statista.com/estadisticas/613361/principales-paisesproductores-de-sal-en-el-mundo/.

[4] Soriano P. y Arturo J., «Estudio técnico para la implementación de un nuevo sistema productivo, para el incremento de la producción en la empresa famovisal s.a",» 2017.

[5] Publimetro, «México tiene la exportadora de sal más grande de mundo.,» 16 febrero 2014. [En línea]. Available: https://www.publimetro.com.mx/mx/noticias/2014/02/16/mexi co-tiene-la-exportadora-de-sal-mas-grande-del-mundo.html

[6] Grupo Industrial ROCHE, «Grupo roche,» [En línea]. Available: http://www.gruporoche.com.mx/historia.html.

[7] FENCOSPA, R.L., «Estadística de producción anual.,» 2017.

[8] FENCOSPA, R.L., «FENCOSPA, R.L.,» [En línea]. Available: https://fencospa.com.pa/antecedentes/.

[9] Google Earth, [En línea]. Available: https://earth.google.com/web/@7.95787708,80.36693247,6.54403548a,5026.09950532d,30.0000412y,0h,0 $\mathrm{t}, 0 \mathrm{r}$.
[10] Milton, «Producción de sal solar de fencospa r.l. Los Santos Panamá» losvideosdemilton1, [en línea]. Available: https://www.youtube.com/watch?v=vAt_f9p35_c.

[11] O. Bernal, «"Proyecto Programación Financiera FENCOSPA, R.L”,» Panamá, 2007.

[12] Walpole, R., Myers, R. y Cruz, R., «"Probabilidad y estadística para ingenieros"» México, 1999.

[13] OPS Panamá, «OPS Panamá» [En línea]. Available: https://www.paho.org/pan/index.php?option=com_content\&vi ew=article $\& i d=1355$ :inteligencia-artificial-la-herramienta-depanama-para-contener-el-covid-19\&Itemid=442. [Último acceso: 31 mayo 2020]. 